\title{
Providing Support for Today's Decision Making Processes
}

\author{
Decision making
}

\author{
Sylvia Encheva* \\ Stord/Haugesund University College \\ Bjørnsonsg. 45, 5528, Haugesund, Norway \\ Polytec, Sørhauggata 128, 5527 Haugesund, Norway \\ *Corresponding author
}

\begin{abstract}
Current decision making processes often involve uncertain information obtained from a number of sources. In addition the available data might be incomplete and/or collected at different time periods, i.e. its significance may require adjustments. In many cases experiments cannot be repeated and conclusions have to be drawn based on opinions of several experts. Working with intervals instead of using crisp values provides an opportunity to accommodate several varieties of data types. This can be done by involvement of grey numbers. Their exact values are unknown but either the corresponding intervals where they possibly belong are known or some sets of numbers where they can be placed are known. Grey numbers are further divided into conceptual, layer, and prediction type according to types of uncertainty they describe. In this work we consider project proposals ranking based on multi criteria multi person decision making. Grey numbers are employed to facilitate handling imprecise data. Concept lattices are further employed for querying in a set of formal concepts extracted from available data. Such processes result in establishing whether a new element belongs to any of the existing pattern structures.
\end{abstract}

Keywords-grey numbers; grey systems; evaluation; uncertainty

\section{INTRODUCTION}

Organizations and firms with reasonably small budgets usually have limited resources for undertaking new projects. Problems are getting even bigger when new proposals have to be considered and subsequently given priority. Decision support systems [10], [12] have been employed to handle selections and rankings in different areas. Some of them are based on methods taken from fuzzy operations, rough sets, soft sets and formal concept analysis, [2].

In research literature persons employed to evaluate attributes, objects, methods, etc. are usually referred to as experts. In some occasions they might very well be. In reality, however, such evaluations are often done by people with different backgrounds, experience, level of expertise, and positions in an organization. They might be in charge of finance, human resources, planning, etc. They have uneven influence on the final outcome and a wide range of preferences. This implies working with imprecise and incomplete data when f. ex. a decision support system is to be employed. Grey numbers and grey systems can handle humans' opinions expressed with uncertainty as well with non crisp values.
Ranking based on multi criteria multi person decision making is in the main focus of this work. Imprecise data is evaluated applying grey numbers.

\section{GREY NUMBERS}

In Grey theory is an effective method used to solve uncertainty problems with discrete data and incomplete information. The theory includes five major parts: grey prediction, grey relational analysis, grey decision, grey programming and grey control, [7], [8], and [11]. A quantitative approach for assessing the qualitative nature of organizational visions is presented in [16].

A grey number is generally denoted by " $\otimes ",[14]$, where a grey number with only a lower bound is denoted by $\otimes \in[a, \infty]$ or $\otimes(\underline{a})$. There are also continuous and discrete grey numbers, black and white numbers, essential and non-essential grey numbers.

A grey system is defined as a system containing uncertain information presented by a grey number and grey variables, [13]. For an initial selection of items to be evaluated we propose use of formal concept analysis [6] and [9]. An information table is to be prepared first relating items and attributes. A concept lattice is to be depicted afterwards and with adequate use of association rules a selection of interesting items can be made. Later on experts' opinions for evaluating different aspects of each of the preselected items are to be gathered. They can be expressed by numerical and linguistic values. Converting linguistic values to numerical intervals can be done by f. ex. following [13], very poor $[0,1]$; poor $[1,3]$; medium poor $[3,4]$; fair $[4,5]$; medium $\operatorname{good}[5,6]$; good $[6$, $9]$; very good $[9,10]$.

The analytic hierarchy process [17] supports decision making where several factors are to be considered. Weight coefficients are attached to factors in order to distinguish their importance. Ranking problems have been previously considered by applying techniques from the analytic hierarchy process [17], [18] as well as mathematical programming methods [19].

Let $P$ be a non-empty ordered set. If $\sup \{x, y\}$ and $\inf \{x, y\}$ exist for all $x, y \in P$, then $P$ is called a lattice, [6]. In a lattice illustrating partial ordering of knowledge values, the logical conjunction is identified with the meet operation and the logical disjunction with the join operation. A context is a triple 
$(G, M, I)$ where $G$ and $M$ are sets and $I \subset G \times M$. The elements of $G$ and $M$ are called objects and attributes respectively, [20].

The rest of this section follows [4] where a query is a new object inserted in a formal context. A concept lattice defines a query space where each formal concept can be considered as a conjunctive Boolean query (i.e. a query where the constraint is given by the conjunction of the attributes in the intent of that formal concept) and a combination of formal concepts provides disjunction and negation. Various relevance measures such as topological distance within the lattice between the query concept and its superconcepts [4] semantic distance [1] extent intersection [15], and Jaccard similarity [3] have been already exploited.

\section{APPLICATIONS}

We consider six proposals $S 1, S 2, S 3, S 4, S 5$, and $S 6$ to be ranked with respect to three attributes: clarity of presentation $Q 1$, quality of proposed outcomes $Q 2$, and a degree to which a proposal supports firm's visions $Q 3$.

Linguistic ratings for attributes and weights are chosen by a committee. Attribute weights and ratings are considered as linguistic variables where

$S=S_{1}, S_{2}, \ldots, S_{m}-$ a discrete set of $m$ possible alternatives,

$Q=Q_{1}, Q_{2}, \ldots, Q_{n} \quad-\quad$ a $\quad$ set of $n$ attributes for assessment,

$\otimes w$ - the vector of attribute weights.

Calculations are done according to the theory presented in [14]. Linguistic ratings for attributes are performed first and calculations of attribute ratings are completed consequently. The weighted normalized decision table is shown in TABLE I.

TABLE I. WEIGHTED NORMALIZED DECISION TABLE

\begin{tabular}{|l|l|l|l|}
\hline Si & \multicolumn{1}{|c|}{ Q1 } & \multicolumn{1}{|c|}{ Q2 } & \multicolumn{1}{|c|}{ Q3 } \\
\hline S1 & {$[0.47,0.95]$} & {$[0.26,0.46]$} & {$[4.5,5.5]$} \\
S2 & {$[0.32,0.45]$} & {$[0.36,0.58]$} & {$[5.25,6.5]$} \\
S3 & {$[0.43,0.55]$} & {$[0.28,0.50]$} & {$[3.0,4.25]$} \\
S4 & {$[0.51,0.63]$} & {$[0.46,0.65]$} & {$[5.5,6.76]$} \\
S5 & {$[0.31,0.52]$} & {$[0.27,0.56]$} & {$[4.78,6.1]$} \\
S6 & {$[0.30,0.51]$} & {$[0.23,0.48]$} & {$[2.19,4.13]$} \\
\hline
\end{tabular}

Thus we can see that the ideal referential alternative is

$S^{\max }=[0.51,0.63],[0.36,0.65],[5.5,6.75], \quad$ and the possibility degrees for $i=1,2,3,4$ are $\mathrm{P}\left\{\mathrm{S}_{\mathrm{i}} \leq \mathrm{S}^{\max }\right\}=$ $\{0.69,0.65,0.74,0.76,0.63,0.62\}$.

The result of ranking is $S 4>S 3>S 1>S 2>S 5>S 6$. According to the performed calculations the fourth proposal is the best choice.

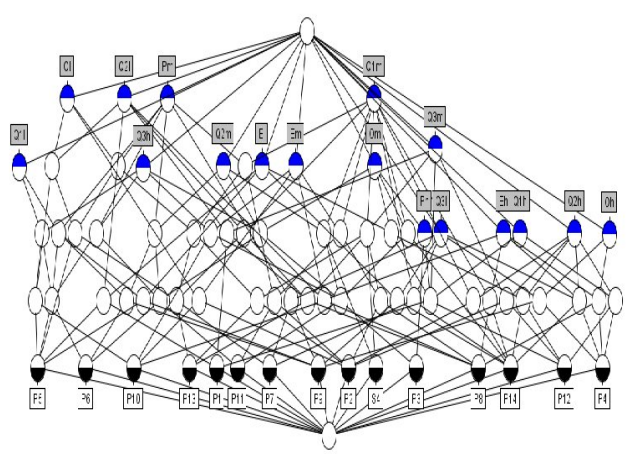

FIGURE I. A LATTICE WHERE THE QUERY IS S4

More often than not differences between several proposals' rankings are marginal. Below we use query [4] from FCA for selecting a proposal satisfying 'requirements' not been part of the 'grey calculations'. Such requirements can be often found among the ones that are difficult to insert in the 'grey calculations'. Suppose there is data related to previous proposals including initial grading, final outcomes in terms of success and failure $O$, need for different expertise $E$, need for new partners $P$, need for additional financial support $F$, project management complications $M$, etc. The remaining job is to find which concepts from the original context are related to the query concept and to what degree.

TABLE II. DATA FROM PREVIOUS PROJECTS

\begin{tabular}{|c|c|c|c|c|c|c|c|c|c|c|c|c|c|c|c|c|c|c|c|c|c|c|c|c|}
\hline & & Q1 & & & Q2 & & & Q3 & & & O & & & $\mathbf{E}$ & & & $\mathbf{P}$ & & & $\mathbf{F}$ & & & $\mathbf{M}$ & \\
\hline & 1 & $\mathrm{~m}$ & h & 1 & $\mathrm{~m}$ & $\mathrm{~h}$ & 1 & $\mathrm{ml}$ & & & $\mathrm{m}$ & & 1 & $\mathrm{~m}$ & & & $\mathrm{~m}$ & h & 1 & $\mathrm{~m}$ & h & 1 & & h \\
\hline P1 & & & $\mathrm{X}$ & $\mathrm{x}$ & & & & $\mathrm{X}$ & & & $\mathrm{X}$ & & $\mathrm{x}$ & & & & & $\mathrm{x}$ & & $\mathrm{x}$ & & & $\mathrm{X}$ & \\
\hline P2 & & $\mathrm{x}$ & & & & $\mathrm{X}$ & & $\mathrm{X}$ & & $\mathrm{x}$ & & & & $\mathrm{x}$ & & & $\mathrm{x}$ & & & & $\mathrm{X}$ & & $\mathrm{x}$ & \\
\hline P3 & & $\mathrm{x}$ & & & $\mathrm{x}$ & & & $x$ & $\mathrm{x}$ & & $\mathrm{X}$ & & & $\mathrm{x}$ & & & & $\mathrm{x}$ & & $\mathrm{x}$ & & $\mathrm{x}$ & & \\
\hline P4 & & & $\mathrm{x}$ & & & $\mathrm{x}$ & $\mathrm{X}$ & & & & & $\mathrm{X}$ & & . & $\mathrm{X}$ & $\mathrm{x}$ & & & $\mathrm{x}$ & & & & & $x$ \\
\hline P5 & $\mathrm{x}$ & & & $\mathrm{x}$ & & & & 2 & $\mathrm{x}$ & $\mathrm{x}$ & & & $\mathrm{x}$ & & & & $\mathrm{x}$ & & & $x$ & & $\mathrm{x}$ & & \\
\hline P6 & $\mathrm{X}$ & & & & $\mathrm{x}$ & & & 2 & $\mathrm{x}$ & & $\mathrm{X}$ & & & 2 & $\mathrm{X}$ & & $\mathrm{x}$ & & & & $\mathrm{X}$ & & & $\mathrm{X}$ \\
\hline P7 & & $\mathrm{x}$ & & $\mathrm{x}$ & & & $\mathrm{X}$ & & & $\mathrm{x}$ & & & & & $\mathrm{X}$ & & $\mathrm{x}$ & & & $x$ & & & $\mathrm{X}$ & \\
\hline P8 & & $\mathrm{x}$ & & $\mathrm{x}$ & & & & $\mathrm{X}$ & & & & $\mathrm{X}$ & & $\mathrm{X}$ & & $\mathrm{X}$ & & & $\mathrm{X}$ & & & $\mathrm{X}$ & & \\
\hline P9 & $\mathrm{x}$ & & & & $\mathrm{x}$ & & $\mathrm{X}$ & & & & & $\mathrm{X}$ & $\mathrm{X}$ & & & & $\mathrm{x}$ & & & & $\mathrm{X}$ & $\mathrm{x}$ & & \\
\hline $\mathrm{P} 10$ & & 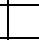 & & $\mathrm{x}$ & & & & $a^{2}$ & $\mathrm{x}$ & $\mathrm{x}$ & & & & $\mathrm{x}$ & & $\mathrm{x}$ & & & & $x$ & & & $\mathrm{X}$ & \\
\hline P11 & & $\mathrm{x}$ & & & $\mathrm{x}$ & & & 2 & $\mathrm{x}$ & & $\mathrm{X}$ & & & $\mathrm{X}$ & & & $\mathrm{x}$ & & $\mathrm{X}$ & & & & $\mathrm{X}$ & \\
\hline P12 & & & $\mathrm{x}$ & & & $\mathrm{X}$ & & $\mathrm{x}$ & & & $\mathrm{X}$ & & $\mathrm{x}$ & & & & & $\mathrm{x}$ & & $\mathrm{x}$ & & & & $\mathrm{x}$ \\
\hline P13 & & $\mathrm{x}$ & & $\mathrm{x}$ & & & & $\mathrm{x}$ & & $\mathrm{x}$ & & & & & $\mathrm{X}$ & & $\mathrm{x}$ & & $\mathrm{x}$ & & & & $\mathrm{X}$ & \\
\hline P14 & & $\mathrm{x}$ & & & & $\mathrm{x}$ & $\mathrm{X}$ & & & & $\mathrm{X}$ & & $\mathrm{x}$ & & & $\mathrm{X}$ & & & & & $\mathrm{X}$ & & & $\mathrm{X}$ \\
\hline S4 & & $\mathrm{x}$ & & & $\mathrm{x}$ & & & $\mathrm{x}$ & & & & & & & & & & & & & & & & \\
\hline S5 & $\mathrm{x}$ & & & & $\mathrm{x}$ & & $\mathrm{x}$ & & & & & & & & & & & & & & & & & \\
\hline
\end{tabular}

At this point is important to consider how to covert number intervals into linguistic values. Dividing f. ex. an interval $[0,1]$ into three subintervals of equal length according to three linguistic values 'low (1), medium (m), high (h)' might not always describe a practical case. Another suggestion can be something like

- 'low - $[0,0.5]$ ',

- 'medium - [0.51, 0.75]', and

- 'high - $[0.76,1]$ '.

The lattice in Figure I. depicted from TABLE I. has proposal S4 as a query. Attributes Q1m and Q2m are shared 
by projects $\mathrm{P} 3$ and $\mathrm{P} 11$ and proposal $\mathrm{S} 4$, i. e. the topological distance between S4 and P3 and P11 with respect to Q1m and $\mathrm{Q} 2 \mathrm{~m}$ is zero. At the same time the topological distance between S4 and P2, P8 and P13 with respect to Q1m and Q3m is zero.

The second lattice depicted from TABLE II. has proposal S5 as a query. The topological distance between S5 and P9 with respect to Q11, Q2m and Q31 is zero. Attributes Q11 and $\mathrm{Q} 2 \mathrm{~m}$ are shared by projects P6 and P9 and proposal S5, while attribute Q31 is shared by projects P4, P9, P14 and proposal S5.

As emphasized in [1] distance measures based on clustering methods are often context-sensitive but they can handle important features which are outside of the reach of traditional statistical measures.

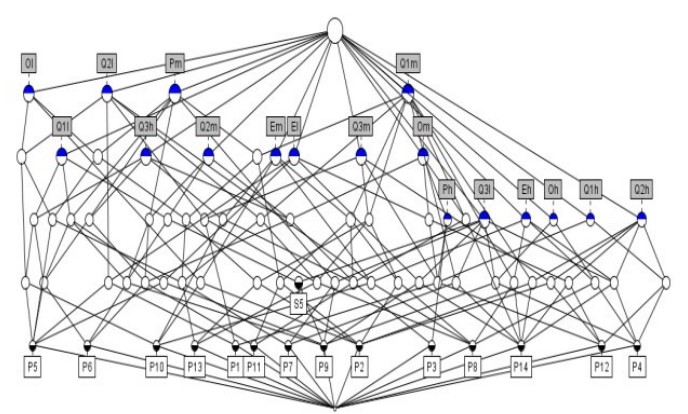

FIGURE II. A LATTICE WHERE THE QUERY IS S5

Based on the available data one can say that proposal S5 is more likely to be in need of additional financial support than proposal S4, while proposal S4 is more likely to be in need of additional expertise than proposal S5. Such indications may affect the final decision on which proposal is to be granted.

As pointed in [1] it is worth investigating the potentials of concept lattices for dealing with ambiguous query terms in query refinement based on the actual content of the collection, [5].

\section{CONCLUSION}

While such an approach can definitely speed up selection processes it has to be kept in mind that different cases require some adjustments. Different organizations come usually with a variety of preferences and interests. All subjective views therefore ought to be incorporated in a decision support system serving a particular organization. Grey system theory is to be further employed for developing a Web based system collecting human opinions and ranking the preselected items.

\section{REFERENCES}

[1] C. Carpineto and G. Romano, "Order theoretical ranking”, Journal of the American Sociaty for Information Science, vol. 51, no. 7, pp. 587-601, 2000 .

[2] C. Carpineto and G. Romano, "Concept Data Analysis: Theory and Applications”, John Wiley and Sons, Ltd., 2004.

[3] V. Codocedo, I. Lykourentzou, H. Astudillo, and A. Napoli, "Using pattern structures to support information retrieval with Formal Concept
Analysis", Proceedings of the International Workshop "What can FCA do for Artificial Intelligence?" Beijing, China, August 5, pp. 15-24, 2013.

[4] J. Cooper and R. Byrd, "Lexical navigation: visually prompted query expansion and refinement", Proceedings of the Second ACM Digital Library Conference, Philadelphia, ACM, pp. 237-246, 1997.

[5] B. A. Davey and H. A. Priestley, "Introduction to lattices and order", Cambridge University Press, Cambridge, 2005.

[6] J. L. Deng, "Control problems of grey systems", System and control letters, vol. 5, pp. 288-294, 1982.

[7] J. L. Deng, "Introduction to grey system theory", Journal of grey systems, vol. 1, pp. 1-24, 1989.

[8] B. Ganter and R. Wille, "Formal Concept Analysis", Springer, 1999.

[9] J. Gettinger, E. Kiesling, C. Stummer, and R. Vetschera, "A comparison of representations for discrete multi-criteria decision problems", Decision Support System, vol. 54, pp. 976-985, 2013.

[10] Y. C. Hu, "Grey relational analysis and radical basis function network for determining costs in learning sequences", Applied mathematics and computation, vol. 184, pp. 291-299, 2007.

[11] J. Huysmans, K. Dejaeger, C. Mues, J. Vanthienen, and B. Baesens, “An empirical evaluation of the comprehensibility of decision table, tree and rule based predictive models", Decision Support Systems, vol. 51, pp. 141-154, 2011.

[12] G. D. Li, D. Yamaguchi, and M. Nagai, "A grey-based decision-making approach to the supplier selection problem", Mathematical and Computer Modelling, vol. 46, pp. 573-581, 2007.

[13] S. Y. Liu and Y. Lin, "Grey Information Theory and Practical Applications", Advanced Information and Knowledge Processing, Springer, 2006.

[14] N. Messai, M. Devignes, A. Napoli, and M. Smail-Tabbone, "Using Domain Knowledge to Guide Lattice-based Complex Data Exploration", In Proceedings of the 2010 conference on ECAI 2010: 19th European Conference on Artificial Intelligence, pp. 847-852, 2010.

[15] F. Rahimnia, M. Moghadasian, and E. Mashregh, "Application of grey theory organizational approach to evaluation of organizational vision”, Grey Systems: Theory and Application, vol. 1, no. 1, pp. 33-46, 2011.

[16] T. L. Saaty and K. Peniwati, "Group Decision Making: Drawing out and Reconciling Differences", Pittsburgh, Pennsylvania: RWS Publications. 2008.

[17] T. L. Saaty, "Principia Mathematica Decernendi: Mathematical Principles of Decision Making", Pittsburgh, Pennsylvania: RWS Publications, 2010.

[18] D. L. Smytka and M. W. Clemens, "Total cost supplier selection problem: a case study", International Journal of purchasing and material management, vol. 29, no. 1, pp. 42-49, 1993.

[19] R. Wille, "Concept lattices and conceptual knowledge systems", Computers Math. Applications, vol. 23, no.(6-9), pp. 493-515, 1992 